\title{
Metabolic re-patterning in COPD airway smooth muscle cells
}

\author{
Charalambos Michaeloudes ${ }^{1,2}$, Chih-Hsi Kuo ${ }^{1,2,3}$, Gulam Haji ${ }^{1,2}$, \\ Donna K. Finch ${ }^{4}$, Andrew J. Halayko, 5,7 , Paul Kirkham ${ }^{8}$, Kian \\ Fan Chung ${ }^{1,2,9}$ and Ian M. Adcock ${ }^{1,2,9}$ on behalf of COPDMAP ${ }^{10}$
}

Affiliations: ${ }^{1}$ Airways Disease, National Heart and Lung Institute, Imperial College London, London, UK. ${ }^{2}$ Biomedical Research Unit, Royal Brompton and Harefield NHS Trust, London, UK. ${ }^{3}$ Dept of Computing and Data Science Institute, Imperial College London, London, UK. ${ }^{4}$ Respiratory, Inflammation and Autoimmunity, Medlmmune Ltd, Cambridge, UK. ${ }^{5}$ Dept of Physiology and Pathophysiology, University of Manitoba, Winnipeg, MB, Canada. ${ }^{6}$ Dept of Internal Medicine, University of Manitoba, Winnipeg, MB, Canada. ${ }^{7}$ Canadian Respiratory Research Network, Ottawa, ON, Canada. ${ }^{8}$ Dept of Biomedical Sciences, Faculty of Science and Engineering, University of Wolverhampton, Wolverhampton, UK. ${ }^{9}$ Both authors contributed equally. ${ }^{10} \mathrm{~A}$ full list of COPDMAP collaborators can be found in the acknowledgements section.

Correspondence: Charalambos Michaeloudes, Airway Disease Section, National Heart and Lung Institute, Imperial College London, Dovehouse Street, London SW3 6LY, UK. E-mail: c.michaeloudes04dimperial.ac.uk

@ERSpublications

A metabolic shift in airway smooth muscle cells of COPD patients may support their increased growth and survival http://ow.ly/XVkb30eUTLJ

Cite this article as: Michaeloudes $\mathrm{C}$, Kuo C-H, Haji G, et al. Metabolic re-patterning in COPD airway smooth muscle cells. Eur Respir J 2017; 50: 1700202 [https://doi.org/10.1183/13993003.00202-2017].

ABSTRACT Chronic obstructive pulmonary disease (COPD) airways are characterised by thickening of airway smooth muscle, partly due to airway smooth muscle cell (ASMC) hyperplasia. Metabolic reprogramming involving increased glycolysis and glutamine catabolism supports the biosynthetic and redox balance required for cellular growth. We examined whether COPD ASMCs show a distinct metabolic phenotype that may contribute to increased growth.

We performed an exploratory intracellular metabolic profile analysis of ASMCs from healthy nonsmokers, healthy smokers and COPD patients, under unstimulated or growth conditions of transforming growth factor (TGF)- $\beta$ and fetal bovine serum (FBS).

COPD ASMCs showed impaired energy balance and accumulation of the glycolytic product lactate, glutamine, fatty acids and amino acids compared to controls in unstimulated and growth conditions. Fatty acid oxidation capacity was reduced under unstimulated conditions. TGF- $\beta$ /FBS-stimulated COPD ASMCs showed restoration of fatty acid oxidation capacity, upregulation of the pentose phosphate pathway product ribose-5-phosphate and of nucleotide biosynthesis intermediates, and increased levels of the glutamine catabolite glutamate. In addition, TGF- $\beta$ /FBS-stimulated COPD ASMCs showed a higher reduced-tooxidised glutathione ratio and lower mitochondrial oxidant levels. Inhibition of glycolysis and glutamine depletion attenuated TGF- $\beta$ /FBS-stimulated growth of COPD ASMCs.

Changes in glycolysis, glutamine and fatty acid metabolism may lead to increased biosynthesis and redox balance, supporting COPD ASMC growth.

This article has supplementary material available from erj.ersjournals.com

Received: Jan 272017 | Accepted after revision: Aug 292017

Conflict of interest: Disclosures can be found alongside this article at erj.ersjournals.com

Copyright OERS 2017. This version is distributed under the terms of the Creative Commons Attribution Licence 4.0. 


\section{Introduction}

Chronic obstructive pulmonary disease (COPD) is characterised by airway remodelling that involves airway smooth muscle thickening, possibly caused by airway smooth muscle cell (ASMC) hypertrophy and/or hyperplasia [1]. ASMC dysfunction is caused, at least in part, by chronic exposure to inflammation-derived mediators, such as transforming growth factor (TGF)- $\beta$ [2]. ASMCs from COPD patients show enhanced proliferation in response to TGF- $\beta$ and fetal bovine serum (FBS), compared to ASMCs from healthy subjects [3]. However, the molecular mechanisms underlining ASMC dysfunction in COPD are not well understood.

Mitochondria are key regulators of metabolism, redox homeostasis and cell survival and proliferation [4]. Impaired mitochondrial function has been demonstrated in the large airways $[5,6]$ and lungs $[7,8]$ of patients with COPD, and may drive lung inflammation and remodelling [6-8]. Importantly, we have shown defective mitochondrial respiration in cultured COPD ASMCs [6]. Mitochondrial dysfunction associated with metabolic changes such as increased glycolysis and glutamine catabolism contribute to aberrant cellular growth in diseases such as pulmonary arterial hypertension (PAH) and cancer [9, 10]. Glycolytic intermediates feed into amino acid and fatty acid synthesis, and into the pentose phosphate pathway (PPP) to produce reduced nicotinamide adenine diphosphate $(\mathrm{NADPH})$ required for redox homeostasis, and ribose-5-phosphate for nucleotide synthesis. Glutamine catabolism provides nitrogen for nucleotide and amino acid synthesis and glutamate for glutathione synthesis [11]. Therefore, these changes support macromolecule synthesis and maintain cellular redox balance, thereby facilitating cell growth and survival.

The metabolomic profile of serum, urine, bronchoalveolar lavage fluid and exhaled breath condensates from COPD patients has been investigated in order to identify novel biomarkers for disease diagnosis and classification [12-20]. However, this approach does not indicate whether a different metabolic profile in lung structural cells, such as ASMCs, contributes to cellular dysfunction in COPD.

We hypothesised that the mitochondrial dysfunction in COPD ASMCs is accompanied by metabolic and redox changes that may contribute to the increased capacity of COPD ASMCs to proliferate. To identify changes in metabolic pathways associated with the hyperproliferative phenotype of COPD ASMCs we investigated the global intracellular metabolome of ASMCs from healthy nonsmokers, healthy smokers and patients with COPD, at baseline and under the growth conditions of TGF- $\beta$ and FBS.

\section{Materials and methods}

Additional details on the methods used in the study are provided in the online supplementary material.

\section{Subject demographics}

ASMCs were isolated from patients with mild/moderate COPD as defined by GOLD criteria, while healthy nonsmokers and healthy smokers, both current and ex-smokers, were used as controls. COPD patients showed significant airflow obstruction, as indicated by the forced expiratory volume in $1 \mathrm{~s}$ (FEV1) and the FEV1/forced vital capacity (FVC) ratio, had no history of asthma, gave a classical history of shortness of breath on exertion and were all smokers. The mean age of COPD patients was significantly higher than that of controls and smoking pack-year history was greater (tables 1 and 2).

\section{ASMC isolation and culture}

ASMCs were isolated from endobronchial biopsies and from second- to fourth-generation segmental airways obtained during lung resection surgery from healthy nonsmoker and healthy smoker subjects and patients with COPD (tables 1 and 2), and placed in culture as described previously [21, 22]. The study was approved by the local ethics committee and informed consent was obtained from all participants.

\section{Untargeted metabolomics analysis}

Following treatment, ASMCs were detached, pelleted by centrifugation and stored at $-80^{\circ} \mathrm{C}$ until processed. Sample preparation and analysis using ultra-high performance liquid chromatography-mass spectrometry or gas chromatography-mass spectrometry was performed by Metabolon (Durham NC, USA), as described previously [23].

Support statement: This study was supported by the MRC-ABPI COPD-MAP consortium (G1001367/1) and a Dunhill Medical Trust grant (R368/0714). It was also supported by the NIHR Respiratory Disease Biomedical Research Unit at the Royal Brompton and Harefield NHS Foundation Trust and Imperial College London. The Canadian Respiratory Research Network (CRRN) is supported by grants from the Canadian Institutes of Health Research (CIHR), Institute of Circulatory and Respiratory Health; Canadian Lung Association (CLA); British Columbia Lung Association; and industry partners Boehringer-Ingelheim Canada, AstraZeneca Canada, Novartis Canada and GlaxoSmithKline. The funders had no role in the study design, data collection and analysis, or preparation of the manuscript. Funding information for this article has been deposited with the Crossref Funder Registry. 
TABLE 1 Clinical characteristics of subjects who provided airway smooth muscle cells (ASMCs) used for metabolomics analysis

\begin{tabular}{lccc} 
& Healthy nonsmokers & Healthy smokers & COPD \\
\hline Subjects & 6 & 6 & 6 \\
Age years & $44.83 \pm 8.63$ & $54.67 \pm 4.15$ & $68.33 \pm 2.32^{*}, \#$ \\
Male/female & $4 / 2$ & $4 / 2$ & $6 / 0$ \\
Smoking (current/ex-smokers) & $\mathrm{NA}$ & $3 / 3$ & $6 / 0$ \\
Smoking pack-years & $\mathrm{NA}$ & $31.67 \pm 5.80$ & $61.20 \pm 11.10$ \\
FEV 1 L & $3.81 \pm 0.41$ & $2.88 \pm 0.22$ & $2.14 \pm 0.22^{*}$ \\
FEV $\%$ predicted & $109.6 \pm 3.28$ & $87.55 \pm 7.54$ & $67.50 \pm 6.97^{* *}$ \\
FVC L & $4.87 \pm 0.50$ & $3.69 \pm 0.30$ & $3.66 \pm 0.18$ \\
FEV 1 FVC $\%$ & $78.17 \pm 2.91$ & $78.28 \pm 1.72$ & $58.39 \pm 5.03^{* *, \# \#}$
\end{tabular}

Data are presented as $\mathrm{n}$ or mean \pm SEM. COPD: chronic obstructive pulmonary disease; FEV1: forced expiratory volume in $1 \mathrm{~s}$; FVC: forced vital capacity; NA: not applicable. ${ }^{*}: p<0.05,{ }^{* *}$ : $p<0.01$ compared to healthy nonsmokers; ${ }^{\#}$ : $p<0.05,{ }^{\# \#}: p<0.01$ compared to healthy smokers.

Determination of differentially expressed metabolites

Data preprocessing and normalisation was performed by Metabolon. Data are presented as "scaled intensity" and were re-scaled to have a median equal to one. Missing values were imputed with the minimum observed value. Differential expression analysis was performed using the Bioconductor $\mathrm{R}$ package limma (http://bioconductor.org/packages/release/bioc/html/limma.html).

\section{Supervised learning algorithm for phenotype classification}

Determination of the optimal number of differentially expressed metabolites was performed using the nearest shrunken centroid method [24], using an algorithm available in the Comprehensive R Archive Network (CRAN-pamr package, https://cran.r-project.org/). Data were adjusted for sex and age using the surrogate variable analysis package in Bioconductor, and principal component analysis was applied.

Pathway analysis

Pathway analysis was performed using the Pathway Activity Profiling algorithm, as previously described [25].

Determination of mitochondrial reactive oxygen species levels

Mitochondrial reactive oxygen species (ROS) levels were determined using the mitochondrial-targeted, redox-sensitive fluorescent probe MitoSOX Red (Invitrogen, Paisley, UK) as previously described [6].

\section{Determination of ASMC proliferation}

Changes in cell proliferation were determined by measuring BrdU incorporation using the Cell Proliferation ELISA kit (Roche Diagnostics, Burgess Hill, UK) according to the manufacturer's

TABLE 2 Clinical characteristics of subjects who provided airway smooth muscle cells (ASMCs) used for the whole study

\begin{tabular}{lccc} 
& Healthy nonsmokers & Healthy smokers & COPD \\
\hline Subjects & 7 & 8 & 8 \\
Age years & $40.71 \pm 6.33$ & $56.50 \pm 3.34$ & $66.63 \pm 2.13^{* *}, \#$ \\
Male/female & $6 / 1$ & $5 / 3$ & $7 / 1$ \\
Smoking (current/ex-smokers) & $\mathrm{NA}$ & $4 / 4$ & $6 / 2$ \\
Smoking pack-years & $\mathrm{NA}$ & $29.25 \pm 4.16$ & $46.00 \pm 8.30$ \\
FEV1 L & $4.22 \pm 0.30$ & $2.68 \pm 0.28^{*}$ & $1.97 \pm 0.21^{* *}$ \\
FEV1 \% predicted & $107.5 \pm 4.33$ & $82.98 \pm 4.98^{*}$ & $62.25 \pm 5.86^{* *}, \#$ \\
FVC L & $5.51 \pm 0.24$ & $3.49 \pm 0.38^{* *}$ & $3.44 \pm 0.25^{* *}$ \\
FEV $/$ FVC \% & $76.40 \pm 3.54$ & $76.91 \pm 1.59$ & $56.71 \pm 3.87^{* *}, \# \#$ \\
\hline
\end{tabular}

Data are presented as $\mathrm{n}$ or mean \pm SEM. COPD: chronic obstructive pulmonary disease; FEV1: forced expiratory volume in $1 \mathrm{~s}$; FVC: forced vital capacity; NA: not applicable. ${ }^{*}: p<0.05,{ }^{* *}: p<0.01$ compared to healthy nonsmokers; ${ }^{\#}: p<0.05,{ }^{\# \# \#}: p<0.001$ compared to healthy smokers. 
instructions. Alternatively, the numbers of live cells were determined by Trypan blue staining and haemocytometer counting.

\section{Statistical analysis}

Statistical analysis was performed using the GraphPad Prism v.5 software (GraphPad Software, San Diego, CA, USA). Unless specified otherwise, intragroup comparisons were performed using the Friedman test followed by Dunn's post hoc test, and intergroup comparisons used the Mann-Whitney test. Correlations were determined using Spearman's correlation coefficient. $\mathrm{p}<0.05$ was considered as statistically significant.

\section{Results}

\section{COPD ASMCS show a distinct metabolic profile}

Metabolomic analysis was performed directly after serum-starvation ( $0 \mathrm{~h}$; baseline) and following $48 \mathrm{~h}$ incubation in the absence (unstimulated) and presence of TGF- $\beta$ /FBS (growth conditions). Under these conditions, COPD ASMCs showed a distinct phenotype compared to ASMCs from healthy smokers, displaying increased proliferation in response to TGF- $\beta /$ FBS, an effect inversely correlated with the subjects' lung function, and a lower $\alpha$-smooth muscle actin mRNA expression (online supplementary figure E1).

Under unstimulated conditions, healthy nonsmoker and healthy smoker samples were separated from COPD samples in principal component (PC) 1 analysis (figure $1 \mathrm{a}$ and b). Following TGF- $\beta / F B S$ treatment, healthy nonsmoker and COPD samples were separated along PC1 (figure $1 \mathrm{c}$ and $\mathrm{d}$ ). The number and identities of differentially regulated metabolites are shown in online supplementary figure E2 and tables E2 and E3. The top differentially regulated metabolic pathways between COPD and healthy nonsmoker and smoker ASMCs included purine and pyrimidine metabolism, amino acid and fatty acid biosynthesis and degradation, pentose and glucuronate interconversions, glutathione metabolism and oxidative phosphorylation (online supplementary tables E4-E7).

\section{Altered energy balance in COPD ASMCs}

ATP levels were not measured in this study; however, the ADP/AMP (figure 2a) and creatine phosphate $(\mathrm{PCr})$ /creatine $(\mathrm{Cr})$ ratios (figure $2 \mathrm{~b}$ ) were reduced, and inorganic phosphate levels were increased (figure 2c) in COPD ASMCs, compared to healthy nonsmoker and smoker ASMCs, under both unstimulated and TGF- $\beta$ /FBS-stimulated conditions. These findings suggest lower ATP levels in COPD ASMCs, both in the absence and presence of mitogenic stimulation.

\section{Altered glucose and nucleotide metabolism in COPD ASMCS}

Glucose levels were not significantly different across the three study groups (figure 3a). No differences were observed in any glycolytic intermediates (data not shown); however, the glycolytic products lactate (figure $3 \mathrm{~b}$ ) and alanine (figure 3c) were significantly increased in COPD ASMCs compared to healthy smoker ASMCs at baseline and after culture under unstimulated and TGF- $\beta$ /FBS-stimulated conditions.

In line with these findings, COPD ASMCs showed reduced baseline mRNA expression of peroxisome proliferator-activated receptor- $\gamma$ coactivator (PGC)- $1 \beta$, a key driver of mitochondrial respiration, and an increase in the baseline mRNA of pyruvate dehydrogenase kinase (PDK)-1, an enzyme that directs pyruvate away from the mitochondrion and towards glycolysis (online supplementary figure E3A-D) [4]. TGF- $\beta$ /FBS stimulation reduced the mRNA of the mitochondrial gene activators PGC- $1 \alpha$ and PGC- $1 \beta$ and increased the glycolytic genes PDK1 and lactate dehydrogenase A in healthy smoker ASMCs. In COPD ASMCs the TGF- $\beta$ /FBS-mediated shift towards glycolytic gene activation was less pronounced, possibly due to their already high baseline glycolytic activity (online supplementary figure E3E-H). These findings suggest a metabolic shift towards glycolysis in COPD ASMCs. The glycolytic inhibitor 2-deoxy-D-glucose (2-DG) reduced TGF- $\beta$ /FBS-induced DNA synthesis in both COPD and healthy smoker ASMCs, suggesting that glycolysis plays a key role in ASMC proliferation (online supplementary figure E3I).

Ribose-5-phosphate levels (figure 3d) were increased in TGF- $\beta$ /FBS-stimulated COPD ASMCs, compared to healthy smoker ASMCs, suggesting an increased flow of glycolytic intermediates through the PPP. In line with this finding, the nucleosides uridine (figure 3e), cytidine (figure 3f), thymidine (figure $3 \mathrm{~g}$ ) and adenosine (figure $3 \mathrm{~h}$ ) were higher in COPD ASMCs compared to healthy nonsmoker and/or healthy smoker ASMCs under TGF- $\beta$ /FBS stimulation. In addition, nucleoside levels in TGF- $\beta /$ FBS-stimulated ASMCs correlated negatively with the FEV1/FVC ratio (online supplementary figure E4A-C). Nucleotide biosynthesis intermediates such as guanosine monophosphate, AMP and uridine monophosphate were also found to be elevated in TGF- $\beta$ /FBS-stimulated COPD ASMCs (online supplementary tables E2 and E3), suggesting that the PPP may support increased nucleotide biosynthesis under growth conditions. 
a)
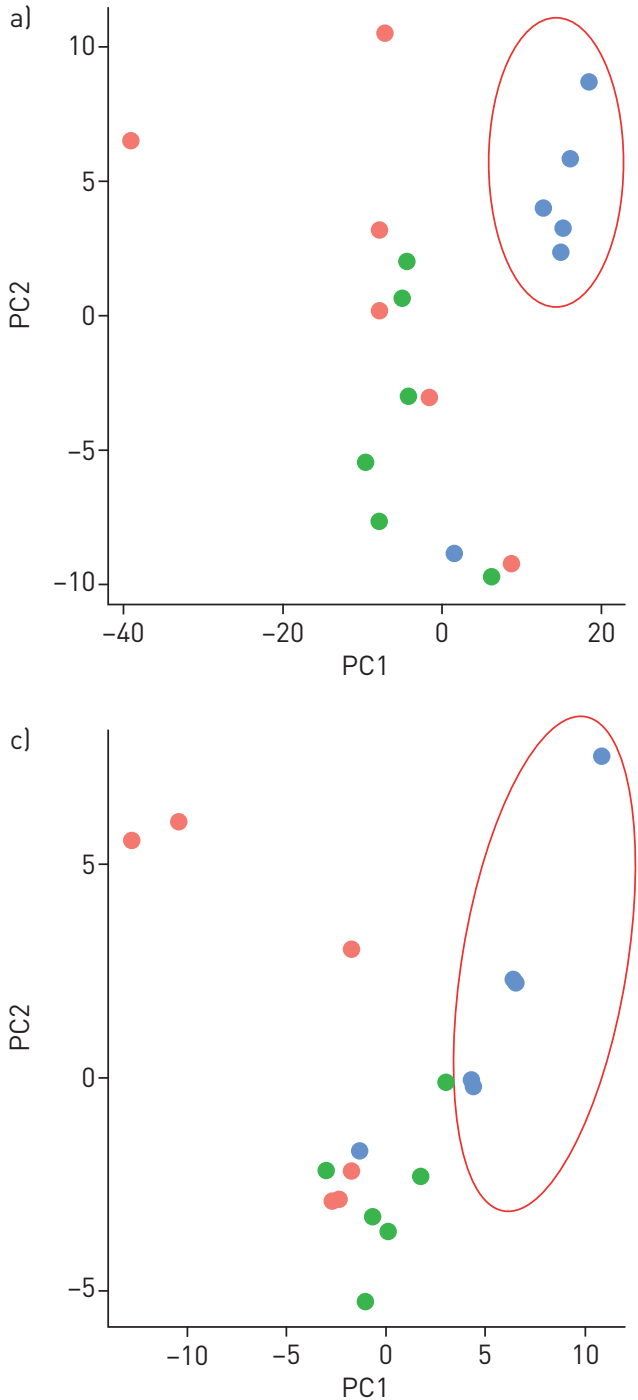

b)

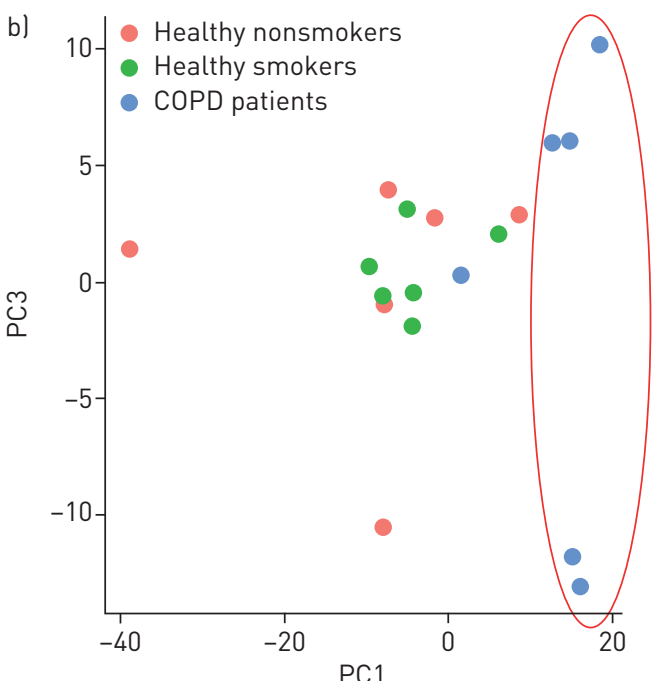

d)

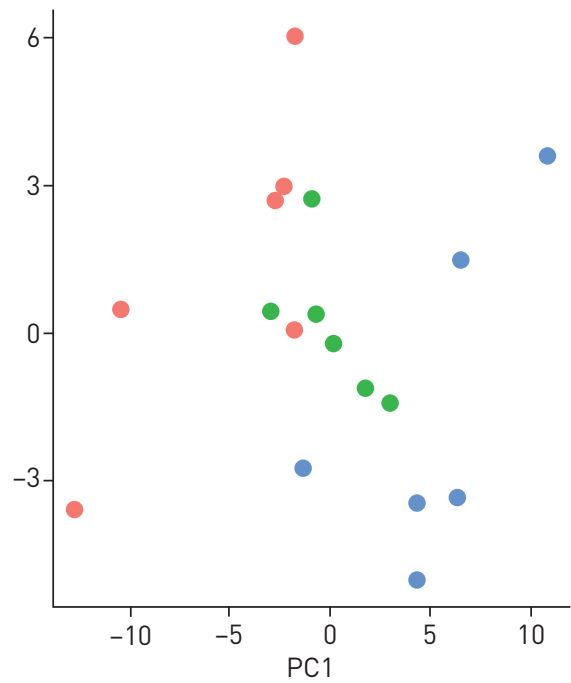

FIGURE 1 Plots of principal component analysis scores along principal component (PC) 1 and PC2 (a and c), or PC1 and PC3 ( $b$ and $d$ ) of differentially expressed metabolites, between chronic obstructive pulmonary disease (COPD) airway smooth muscle cell (ASMCs) and healthy nonsmoker and healthy smoker ASMCs, under unstimulated ( $a$ and b) and transforming growth factor- $\beta /$ fetal bovine serum-stimulated ( $c$ and $d$ ) conditions.

\section{Altered glutamine metabolism in COPD ASMCs}

Glutamine levels at baseline and after culture in the absence or presence of TGF- $\beta / F B S$ were increased in COPD ASMCs compared to healthy smokers and nonsmokers (figure 4a), and negatively correlated with the $\mathrm{FEV} 1 / \mathrm{FVC}$ ratio (online supplementary figure E5A-C). Glutamate (figure $4 \mathrm{~b}$ ) and $\gamma$-aminobutyrate (figure $4 \mathrm{c}$ ), a glutamate metabolite, were significantly increased in TGF- $\beta / F B S-$ treated COPD ASMCs compared to healthy nonsmokers, suggesting increased glutamine catabolism under growth conditions. Glutamine depletion partially attenuated TGF- $\beta$ /FBS-induced DNA synthesis, suggesting a role of glutamine metabolism in ASMC proliferation (online supplementary figure E5D).

\section{Altered fatty acid and amino acid metabolism in COPD ASMCS}

Most of the medium- and long-chain fatty acids (online supplementary table E8) detected, including caproate (figure 5a), myristoleate (figure 5b), caprylate (figure $5 \mathrm{c}$ ) and vaccenate (figure $5 \mathrm{~d}$ ), were increased after culture under unstimulated and TGF- $\beta / F B S$-stimulated conditions in COPD ASMCs, compared to healthy nonsmokers and/or healthy smokers, indicating increased fatty acid synthesis or uptake. The ratios of acetylcarnitine (C2) to free carnitine (C0) (figure 5e) and the sum of $\mathrm{C} 2$ and propionylcarnitine $(\mathrm{C} 3)$ to free carnitine $((\mathrm{C} 2+\mathrm{C} 3) / \mathrm{C} 0)$ (figure $5 \mathrm{f})$, indices of fatty acid oxidation capacity and hexanoylcarnitine (C6) (figure 5g) levels were all reduced at baseline and under unstimulated 

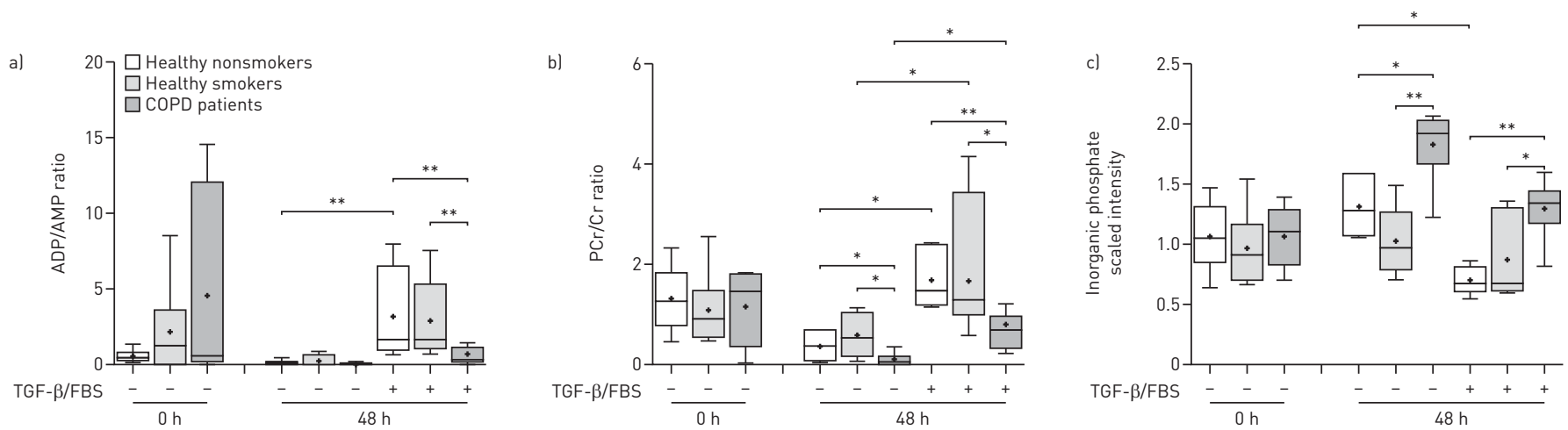

FIGURE 2 Relative ADP/AMP and creatine phosphate (PCr)/creatine $(\mathrm{Cr})$ ratios and inorganic phosphate levels. Airway smooth muscle cells isolated from healthy nonsmokers $(n=6)$, healthy smokers $(n=6)$ and patients with chronic obstructive pulmonary disease (COPD) (n=6) were serum-starved overnight. Cell pellets were collected immediately after starvation $(\mathrm{t}=0)$ or after incubation in the absence or presence of transforming growth factor (TGF)- $\beta$ (1 $\left.\mathrm{ng} \cdot \mathrm{mL}^{-1}\right)$ and fetal bovine serum (FBS) $(2.5 \%)$ for $48 \mathrm{~h}$. The ratios of the scaled intensities of a) ADP/AMP and b) $\mathrm{PCr} / \mathrm{Cr}$, and the scaled intensities of cl inorganic phosphate were determined in cell lysates using liquid chromatography mass spectrometry. Whiskers represent the spread of the data points; horizontal lines indicate the median value; and the + symbols indicate the mean of the values. ${ }^{*}: p<0.05 ; * *: p<0.01$.

conditions in healthy smoker and COPD ASMCs compared to healthy nonsmoker cells. In addition, the baseline $\mathrm{C} 2 / \mathrm{C} 0$ ratio (figure $5 \mathrm{e}$ ) and $\mathrm{C} 6$ (figure $5 \mathrm{~g}$ ) levels, and the $(\mathrm{C} 2+\mathrm{C} 3) / \mathrm{C} 0$ ratio in unstimulated cells (figure 5f) were lower in COPD ASMCs compared to healthy smoker ASMCs.

The $(\mathrm{C} 2+\mathrm{C} 3) / \mathrm{C} 0$ ratios in untreated ASMCs correlated positively with the $\mathrm{FEV} 1 / \mathrm{FVC}$ ratio and correlated negatively with age, suggesting an association of attenuated fatty acid oxidation with lung dysfunction and increasing age (online supplementary figure E6A-B). TGF- $\beta / F B S$ restored the C2/C0 (figure 5e) and (C2 $+\mathrm{C} 3$ )/C0 (figure 5f) ratios and hexanoylcarnitine (figure 5g) levels in healthy smoker and COPD ASMCs, suggesting an increase in fatty acid oxidation during proliferation.

\section{Enhanced glutathione biosynthesis and reduced mitochondrial ROS levels in COPD ASMCs}

The ratio of reduced (GSH) to oxidised (GSSG) glutathione was similar between the study groups at baseline. However, after culture under unstimulated conditions, the GSH/GSSG ratio was lower in healthy smoker ASMCs and showed a statistically nonsignificant reduction in COPD ASMCs (figure 6a), reflecting oxidant-antioxidant imbalance in healthy smoker and COPD cells in the absence of mitogenic stimulation. In contrast, the GSH/GSSG ratio in TGF- $\beta$ /FBS-treated COPD ASMCs was higher compared to healthy nonsmoker ASMCs, while healthy smoker ASMCs showed an increasing trend (figure 6a).

In addition, TGF- $\beta$ /FBS-treated COPD ASMCs had significantly lower mitochondrial ROS levels, and healthy smoker ASMCs showed a trend towards reduced levels, compared to healthy nonsmoker ASMCs (figure 6b). The glutathione synthesis inhibitor buthionine sulfoximine $(10-25 \mu \mathrm{M})$ increased mitochondrial ROS levels both in the absence and presence of TGF- $\beta /$ FBS (online supplementary figure E7A), and inhibited the increase in COPD ASMC number in response to TGF- $\beta / F B S$ (online supplementary figure E7B). Thus, COPD, and to a lesser extent, healthy smoker ASMCs show improved redox homeostasis under growth conditions, which may contribute to their increased survival and proliferation.

\section{Discussion}

We have demonstrated that COPD ASMCs exhibit a hyperproliferative phenotype associated with an altered metabolic profile in vitro. COPD cells show lower ATP levels, indicated by lower ADP/AMP and $\mathrm{PCr} / \mathrm{Cr}$ ratios, both under unstimulated and growth conditions. In addition, fatty acid oxidation capacity was reduced in COPD ASMCs compared to healthy nonsmoker and smoker ASMCs under unstimulated conditions, but it was restored under growth conditions. COPD ASMCs showed increased levels of glutamine and the glycolytic products lactate and alanine, compared to healthy nonsmoker and/or smoker ASMCs, under both unstimulated and growth conditions. Additionally, TGF- $\beta$ /FBS-stimulated COPD ASMCs showed higher levels of ribose-5-phosphate, indicating increased flow of glycolytic intermediates through the PPP, and accumulation of glutamine catabolites. Glycolysis, PPP and glutamine catabolism generate intermediates required for the biosynthesis of macromolecules and the maintenance of redox balance [11]. Indeed, fatty acid and amino acid levels were elevated in COPD ASMCs compared to healthy nonsmoker and/or smoker cells under unstimulated and growth conditions. Moreover, TGF- $\beta /$ FBS-stimulated COPD ASMCs maintained higher levels of nucleotide biosynthesis intermediates, and a 

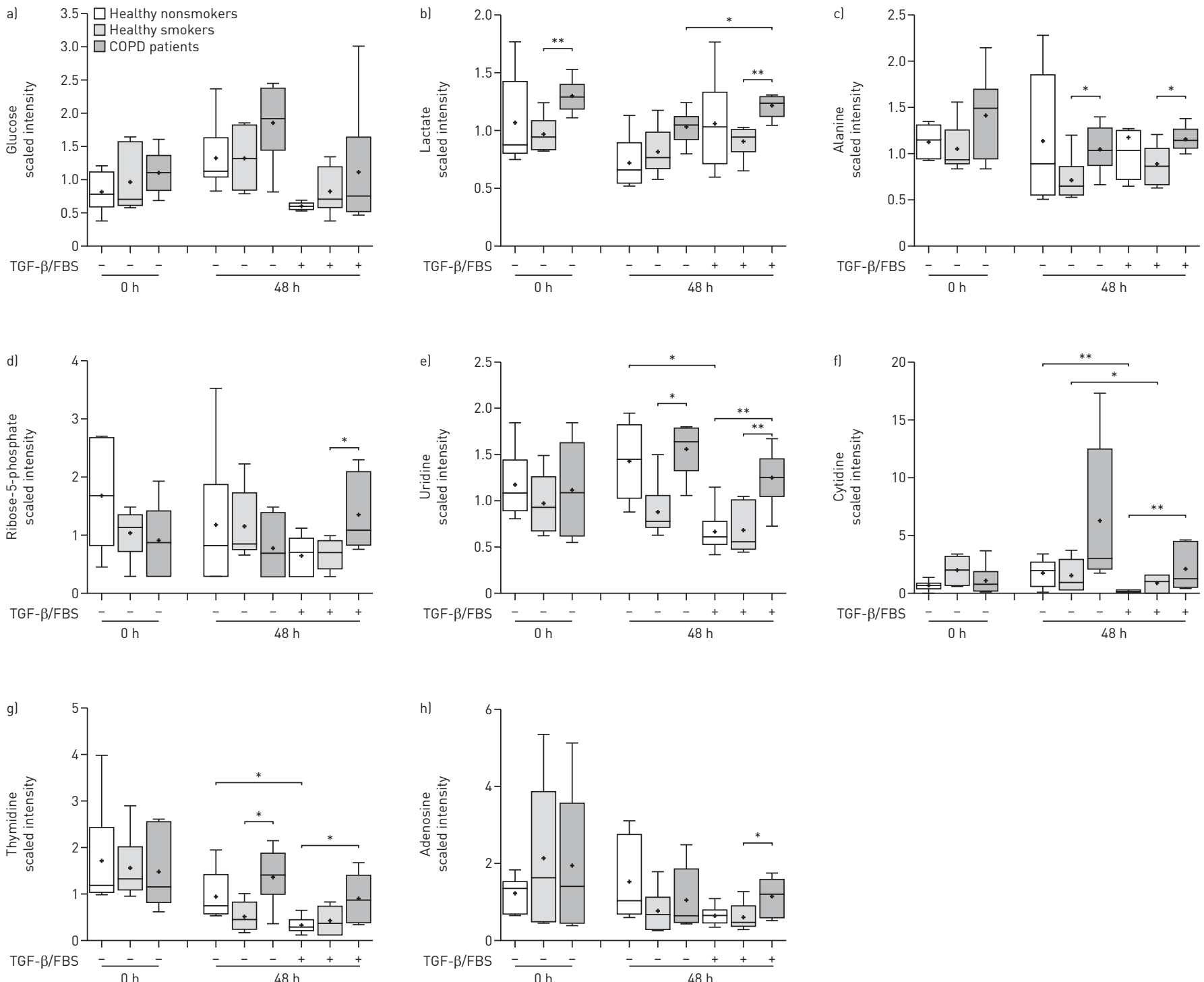

FIGURE 3 Relative levels of metabolites of glycolysis, pentose phosphate pathway and nucleotide metabolism. Airway smooth muscle cells isolated from healthy nonsmokers $(n=6)$, healthy smokers $(n=6)$ and patients with chronic obstructive pulmonary disease (COPD) (n=6) were serum-starved overnight. Cell pellets were collected immediately after starvation $(t=0)$ or after incubation in the absence or presence of transforming growth factor (TGF)- $\beta\left(1 \mathrm{ng} \cdot \mathrm{mL}^{-1}\right)$ and fetal bovine serum (FBS) $(2.5 \%)$ for $48 \mathrm{~h}$. The scaled intensities of a) glucose, b) lactate, c) alanine, d) ribose-5-phosphate, e) uridine, f) cytidine, gl thymidine and h) adenosine were determined in cell lysates by liquid chromatography mass spectrometry or gas chromatography mass spectrometry. Whiskers represent the spread of the data points; horizontal lines indicate the median value; and the + symbols indicate the mean of the values. ${ }^{*}: p<0.05 ;{ }^{* *}: p<0.01$.

higher reduced to oxidised glutathione ratio and lower mitochondrial oxidant levels. Increased availability of macromolecules and maintenance of redox balance may support increased proliferation in COPD ASMCs.

Low ADP/AMP and $\mathrm{PCr} / \mathrm{Cr}$ ratios and elevated inorganic phosphate levels indicate lower ATP levels in COPD ASMCs both in the absence and presence of mitogenic stimulation. This possibly reflects a reduction in mitochondrial respiration in COPD ASMCs, as previously described [6], and is consistent with a lower PGC-1 $\beta$ mRNA expression. Fatty acids interact with carnitine molecules, forming long-chain acylcarnitines that transport fatty acids to the mitochondrion and peroxisomes where they undergo fatty acid oxidation to produce acetyl-coenzyme $\mathrm{A}, \mathrm{NADH}$ and $\mathrm{FADH}_{2}$ required for mitochondrial respiration [26]. Decreased baseline ratios of even-numbered $(\mathrm{C} 2)$ and total $(\mathrm{C} 2+\mathrm{C} 3)$ acylcarnitines to free carnitine $(\mathrm{C} 0)$ suggest an impaired fatty acid oxidation capacity in COPD ASMCs, which may also contribute to the attenuated mitochondrial respiration. Carnitine levels are reduced in an elastase-induced mouse model of emphysema [27], while impaired fatty acid oxidation and lipid accumulation have been reported in ageing mice [28]. The accumulation of fatty acids observed in COPD ASMCs under unstimulated and growth 

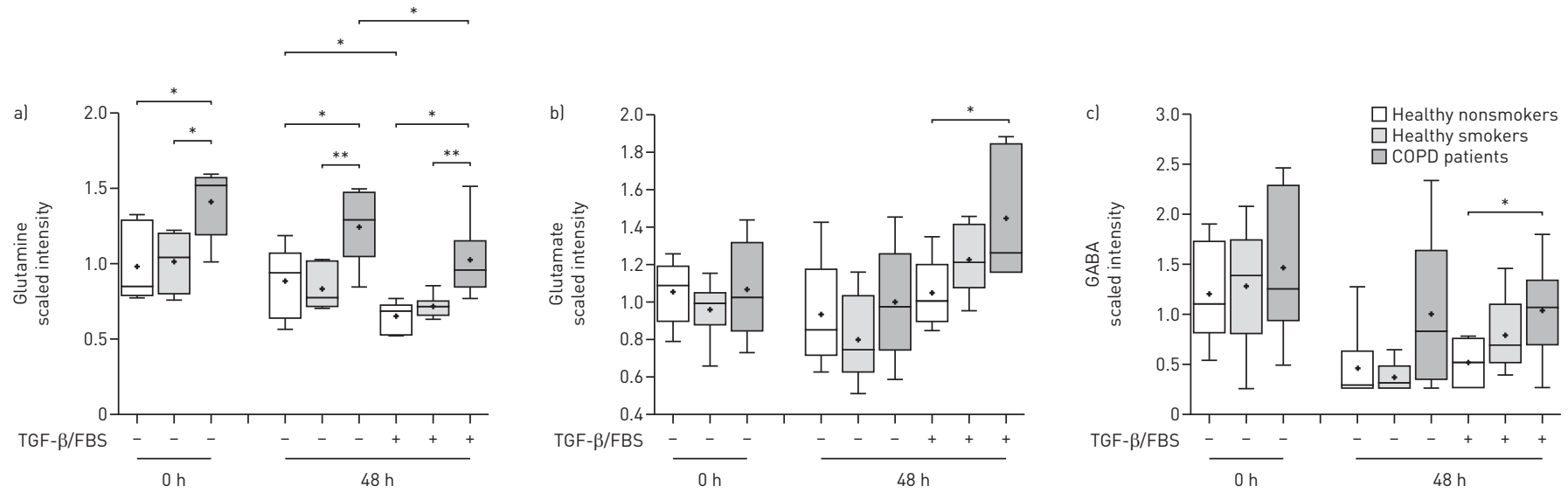

FIGURE 4 Relative levels of glutamine and glutamine catabolites. Airway smooth muscle cells isolated from healthy nonsmokers (n=6), healthy smokers $(n=6)$ and patients with chronic obstructive pulmonary disease (COPD) $(n=6)$ were serum-starved overnight. Cell pellets were collected immediately after starvation ( $\mathrm{t}=0)$ or after incubation in the absence or presence of transforming growth factor (TGF)- $\beta$ (1 ng.mL $\mathrm{mL}^{-1}$ ) and fetal bovine serum (FBS) (2.5\%) for $48 \mathrm{~h}$. The scaled intensities of a) glutamine, b) glutamate and c) $\gamma$-aminobutyrate (GABA) were determined in cell lysates by liquid chromatography mass spectrometry or gas chromatography mass spectrometry. Whiskers represent the spread of the data points; horizontal lines indicate the median value; and the + symbols indicate the mean of the values. ${ }^{*}: p<0.05 ;{ }^{* *}: p<0.01$.

conditions may result from reduced fatty acid oxidation and/or increased uptake or biosynthesis of fatty acid in these cells.

Lactate, alanine and glutamine levels are elevated in COPD ASMCs, suggesting increased glycolytic activity and increased glutamine uptake or biosynthesis. COPD ASMCs showed elevated baseline mRNA expression of PDK1, which mediates the redirection of pyruvate towards lactate and alanine production [11], suggesting that the glycolytic shift possibly occurs downstream of pyruvate. This may explain our observation that COPD and healthy smoker ASMCs had the same sensitivity to the antiproliferative effect of 2-DG, an inhibitor of the first step of glycolysis [11]. Increased use of glycolysis and glutamine for energy production may be an adaptive response to mitochondrial dysfunction [9]. Reduced mitochondrial respiration in cigarette smoke extract-exposed lung epithelial cells has been shown to be associated with a shift towards glycolysis [29]. Glycolysis and glutamine catabolism support hyperproliferation and survival in cancer cells by providing precursors for biosynthesis and antioxidant protection. Glycolytic intermediates feed into fatty acid and amino acid biosynthesis, and into the PPP to generate ribose-5-phosphate for nucleotide synthesis, and NADPH to maintain redox balance [30]. Glutamine is catabolised to glutamate, donating its amide nitrogen for nucleotide synthesis. In addition, glutamate feeds into the Kreb's cycle through its conversion to $\alpha$-ketoglutarate leading to the production of NADPH and lactate, and acts as a precursor for glutathione synthesis [11].

In addition to elevated fatty acid levels, COPD ASMCs showed an increase in the majority of amino acids (online supplementary table E9) under both unstimulated and growth conditions. This increased availability of fatty acid and amino acids may be a result of increased biosynthesis; however, autophagy may also contribute to this effect [31]. Moreover, under growth conditions COPD ASMCs showed evidence of enhanced nucleotide biosynthesis and augmented antioxidant protection, reflected by a higher GSH/GSSG ratio and lower mitochondrial ROS levels. The increased PPP activity and glutamine catabolism observed in COPD ASMCs under growth conditions possibly drives these processes through the production of nucleotide precursors and NADPH. Enhanced glutathione biosynthesis may also be involved in the enhanced antioxidant response. Glutamate, a constituent of glutathione, and S-adenosyl methionine and cystathionine intermediates of the methionine cycle and transulfuration [32], which provide cysteine for glutathione synthesis, are increased in TGF- $\beta / F B S$-stimulated COPD ASMCs (online supplementary figure E7C-D).

COPD ASMCs show evidence of reduced mitochondrial respiration accompanied by increased glycolysis and glutamine utilisation, processes that support biosynthesis and antioxidant responses. The greater availability of biosynthetic intermediates and antioxidant protection may help drive the associated enhanced proliferation seen in COPD cells [3]. A similar metabolic phenotype, involving reduced mitochondrial respiration and increased glycolysis, PPP activity and glutamine utilisation, associated with increased biosynthetic activity, has been shown to contribute to increased vascular smooth muscle cell and endothelial cell growth in PAH [33-35]. Thus, the metabolic reprogramming observed in COPD ASMCs may contribute to their hyperproliferative phenotype. This is supported by our findings showing 
a)
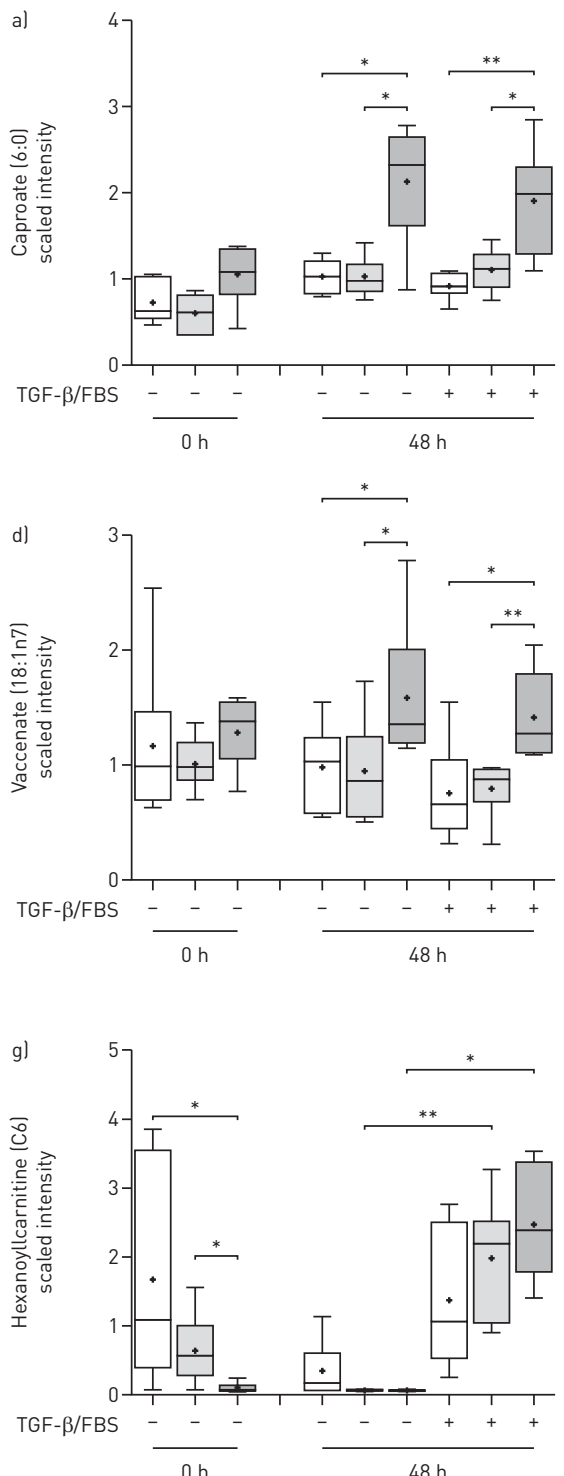

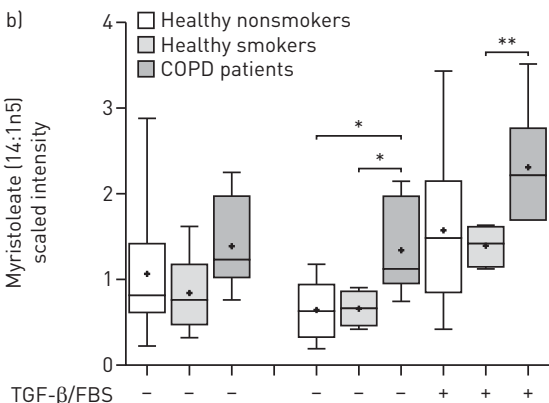

TGF-B/FBS

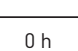

$48 \mathrm{~h}$

e)

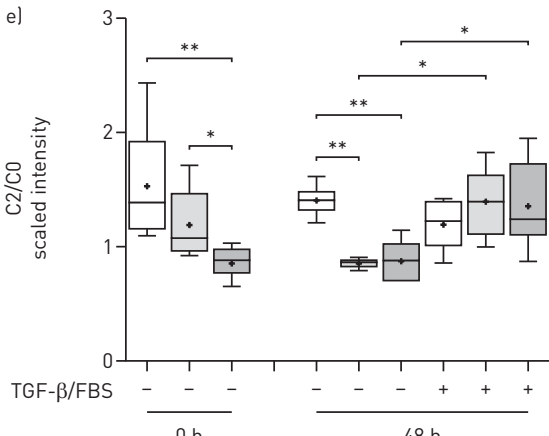

$\mathrm{Oh}$

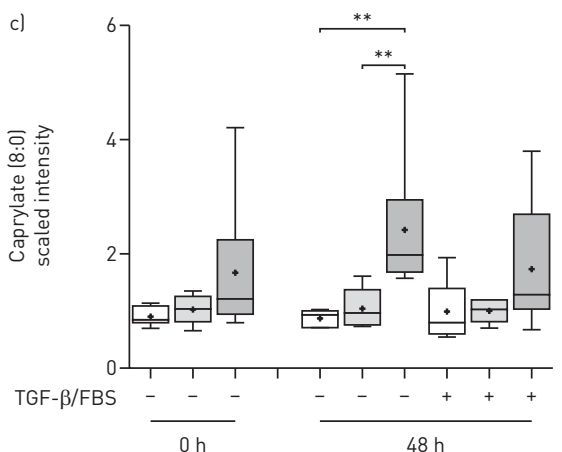

f)

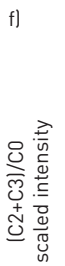

TGF- $\beta$ /FB

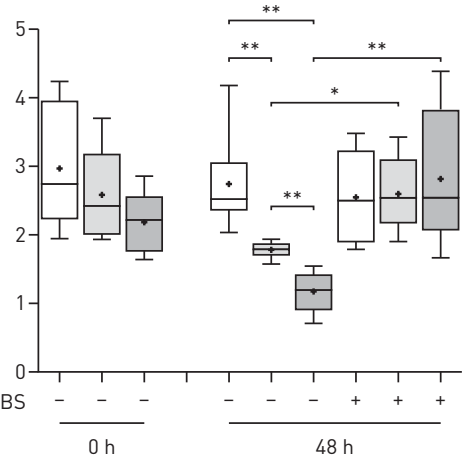

FIGURE 5 Relative levels of fatty acids and intermediates of carnitine metabolism. Airway smooth muscle cells (ASMCs) isolated from healthy nonsmokers $(n=6)$, healthy smokers $(n=6)$ and patients with chronic obstructive pulmonary disease (COPD) ( $=6$ ) were serum-starved overnight. Cell pellets were collected immediately after starvation ( $t=0$ ) or after incubation in the absence or presence of transforming growth factor (TGF)- $\beta$ $\left(1 \mathrm{ng} \cdot \mathrm{mL}^{-1}\right)$ and fetal bovine serum (FBS) (2.5\%) for $48 \mathrm{~h}$. The scaled intensities of a) caproate, b) myristoleate, c) caprylate, d) vaccenate and g) hexanoylcarnitine, as well as the e) ratio of acetylcarnitine (C2) to free carnitine (C0) and f) the ratio of the sum of $\mathrm{C} 2$ and propionylcarnitine (C3) to free carnitine (CO) were determined in cell lysates by liquid chromatography mass spectrometry or gas chromatography mass spectrometry. Whiskers represent the spread of the data points; horizontal lines indicate the median value; and the + symbols indicate the mean of the values. ${ }^{*}: p<0.05 ;{ }^{* *}: p<0.01$.

attenuation of TGF- $\beta$ /FBS-mediated COPD ASMC proliferation by glycolysis and glutathione synthesis inhibition, and glutamine depletion. These mechanisms merit further investigation.

The molecular mechanisms underlying the metabolic shift in COPD ASMCs are currently unknown. In line with our findings, studies in COPD lung tissue and airway epithelial cells have reported downregulation of genes involved in mitochondrial function, including oxidative phosphorylation, and increased expression of genes involved in glycolysis, PPP and glutathione synthesis [36-38]. Prolonged exposure to cigarette smoke may play a role in these changes as ASMCs from healthy smokers show distinct metabolic differences such as in fatty acid oxidation and methionine metabolism compared to healthy nonsmokers. Metabolic reprogramming is known to be driven by mitochondrial dysfunction and pathways such as the PI3K/Akt, mTOR and hypoxia-inducible factor- $1 \alpha$, which play a key role in COPD pathogenesis $[10,11,39]$. We cannot exclude the possibility that some of these changes may be epiphenomena rather than direct causes of the aberrant phenotype of COPD ASMCs. Future studies will 

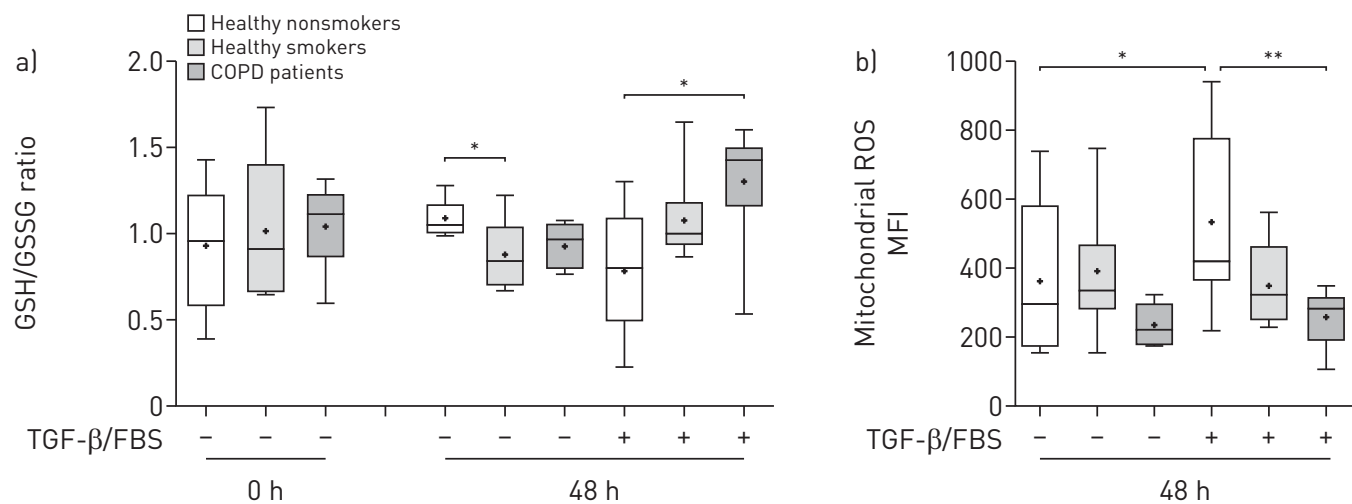

FIGURE 6 Relative ratios of reduced/oxidised glutathione and mitochondrial reactive oxygen species (ROS) levels. a) Airway smooth muscle cells (ASMCs) isolated from healthy nonsmokers $(n=6)$, healthy smokers $(n=6)$ and patients with chronic obstructive pulmonary disease (COPD) $(n=6)$ were serum-starved overnight Cell pellets were collected immediately after starvation $(t=0)$ or after incubation in the absence or presence of transforming growth factor (TGF)- $\beta\left(1 \mathrm{ng} \cdot \mathrm{mL}^{-1}\right)$ and fetal bovine serum (FBS) $(2.5 \%)$ for $48 \mathrm{~h}$. The ratio of the scaled intensities of reduced to oxidised glutathione (GSH/GSSG) was determined in cell lysates by liquid chromatography mass spectrometry; b) ASMCs isolated from healthy nonsmokers ( $n=7)$, healthy smokers $(n=8)$ and patients with COPD $(n=8)$ were serum-starved overnight and incubated in the absence or presence of TGF- $\beta$ /FBS for $48 \mathrm{~h}$. Mitochondrial ROS levels were determined using MitoSOX (Invitrogen, Paisley, UK) staining and expressed as median fluorescence intensity (MFI). Whiskers represent the spread of the data points; horizontal lines indicate the median value; and the + symbols indicate the mean of the values. $*$ : $p<0.05 ; * *$ : $p<0.01$

aim to validate and elucidate these mechanisms and investigate their possible role as drivers of the defective airway smooth muscle function in COPD.

A limitation of our study is the limited number of subjects. Nevertheless, in this preliminary study, we were able to show significant differences in the metabolomic profile of COPD ASMCs. Another limitation is the higher mean age of the COPD patients, which may be a confounding factor in our study, as age is associated with impaired cellular metabolic activity [40]. We cannot exclude the possibility that some of the metabolic changes we observe in COPD ASMCs are age-related; however, age cannot entirely explain the differences we observed between COPD and controls.

In conclusion, we demonstrate that COPD ASMCs demonstrate a distinct metabolic and redox profile compared to those from healthy nonsmokers and smokers. This involves a shift in glucose and glutamine metabolism that may support increased biosynthesis and enhanced antioxidant levels. These metabolic changes are associated with increased cellular growth, and thus may be molecular targets for reversing airway smooth muscle dysfunction in COPD.

\section{Acknowledgements}

The COPDMAP collaborators are as follows. Peter J. Barnes, Airways Disease Division, National Heart and Lung Institute, Imperial College London, London, UK; Christopher E. Brightling, Institute of Lung Health, Dept of Infection, Inflammation and Immunity, University of Leicester, Leicester, UK; Donna E. Davies, The Brooke Laboratories, Division of Infection, Inflammation and Repair, School of Medicine, University of Southampton, Southampton General Hospital, Southampton, UK; Andrew J. Fisher, Institute of Cellular Medicine, Newcastle University, and Cardiopulmonary Transplantation, Institute of Transplantation, Freeman Hospital, Newcastle upon Tyne, UK; Alasdair Gaw, Innovate UK, Technology Strategy Board, Swindon, UK; Alan J. Knox, Centre for Respiratory Research, City Hospital, Nottingham, UK; Ruth J. Mayer, GlaxoSmithKline, King of Prussia, PA, USA; Michael Polkey, NIHR Respiratory Biomedical Research Unit, Royal Brompton and Harefield NHS Foundation Trust and Imperial College London, London; Michael Salmon, Biology Discovery, Merck Research Laboratories, Boston, MA, USA; Yolanda Sanchez, GlaxoSmithKline; Dave Singh, University of Manchester, Medicines Evaluation Unit, University Hospital of South Manchester NHS Foundation Trust, Manchester, UK; Ruth Tal-Singer, GlaxoSmithKline.

\section{References}

1 Chung KF. The role of airway smooth muscle in the pathogenesis of airway wall remodeling in chronic obstructive pulmonary disease. Proc Am Thorac Soc 2005; 2: 347-354.

2 Ichimaru Y, Krimmer DI, Burgess JK, et al. TGF- $\beta$ enhances deposition of perlecan from COPD airway smooth muscle. Am J Physiol Lung Cell Mol Physiol 2012; 302: L325-L333.

3 Perry M, Baker J, Chung KF. Airway smooth muscle cells from patients with COPD exhibit a higher degree of cellular proliferation and steroid insensitivity than that from healthy patients. Eur Respir J 2011; 38: Suppl. 55, 748.

4 McBride HM, Neuspiel M, Wasiak S. Mitochondria: more than just a powerhouse. Curr Biol 2006; 16: R551-R560. 
5 Hoffmann RF, Zarrintan S, Brandenburg SM, et al. Prolonged cigarette smoke exposure alters mitochondria structure and function in airway epithelial cells. Respir Res 2013; 14: 97.

6 Wiegman $\mathrm{CH}$, Michaeloudes C, Haji G, et al. Oxidative stress-induced mitochondrial dysfunction drives inflammation and airway smooth muscle remodeling in patients with chronic obstructive pulmonary disease. J Allergy Clin Immunol 2015; 136: 769-780.

7 Ahmad T, Sundar IK, Lerner CA, et al. Impaired mitophagy leads to cigarette smoke stress-induced cellular senescence: implications for chronic obstructive pulmonary disease. FASEB J 2015; 29: 2912-2929.

8 Mizumura K, Cloonan SM, Nakahira K, et al. Mitophagy-dependent necroptosis contributes to the pathogenesis of COPD. J Clin Invest 2014; 124: 3987-4003.

9 Sutendra G, Bonnet S, Rochefort G, et al. Fatty acid oxidation and malonyl-CoA decarboxylase in the vascular remodeling of pulmonary hypertension. Sci Transl Med 2010; 2: 44ra58.

10 Srinivasan S, Guha M, Dong DW, et al. Disruption of cytochrome c oxidase function induces the Warburg effect and metabolic reprogramming. Oncogene 2016; 35: 1585-1595.

11 Tennant DA, Durán RV, Gottlieb E. Targeting metabolic transformation for cancer therapy. Nat Rev Cancer 2010; 10: $267-277$.

12 Adamko DJ, Nair P, Mayers I, et al. Metabolomic profiling of asthma and chronic obstructive pulmonary disease: a pilot study differentiating diseases. J Allergy Clin Immunol 2015; 136: 571-580.

13 Basanta M, Jarvis RM, Xu Y, et al. Non-invasive metabolomic analysis of breath using differential mobility spectrometry in patients with chronic obstructive pulmonary disease and healthy smokers. Analyst 2010; 135: 315-320.

14 Bowler RP, Jacobson S, Cruickshank C, et al. Plasma sphingolipids associated with chronic obstructive pulmonary disease phenotypes. Am J Respir Crit Care Med 2015; 191: 275-284.

15 Ubhi BK, Cheng KK, Dong J, et al. Targeted metabolomics identifies perturbations in amino acid metabolism that sub-classify patients with COPD. Mol Biosyst 2012; 8: 3125-3133.

16 Ubhi BK, Riley JH, Shaw PA, et al. Metabolic profiling detects biomarkers of protein degradation in COPD patients. Eur Respir J 2012; 40: 345-355.

17 Wang L, Tang Y, Liu S, et al. Metabonomic profiling of serum and urine by ${ }^{1} \mathrm{H}$ NMR-based spectroscopy discriminates patients with chronic obstructive pulmonary disease and healthy individuals. PLoS One 2013; 8: e65675.

18 Motta A, Paris D, Melck D, et al. Nuclear magnetic resonance-based metabolomics of exhaled breath condensate: methodological aspects. Eur Respir J 2012; 39: 498-500.

19 Sharma P, Tran T, Stelmack GL, et al. Expression of the dystrophin-glycoprotein complex is a marker for human airway smooth muscle phenotype maturation. Am J Physiol Lung Cell Mol Physiol 2008; 294: L57-L68.

20 Naz S, Kolmert J, Yang M, et al. Metabolomics analysis identifies sex-associated metabotypes of oxidative stress and the autotaxin-lysoPA axis in COPD. Eur Respir J 2017; 49: 1602322

21 Michaeloudes C, Chang PJ, Petrou M, et al. Transforming growth factor- $\beta$ and nuclear factor E2-related factor 2 regulate antioxidant responses in airway smooth muscle cells: role in asthma. Am J Respir Crit Care Med 2011; 184: 894-903.

22 Gosens R, Stelmack GL, Dueck G, et al. Caveolae facilitate muscarinic receptor-mediated intracellular Ca ${ }^{2+}$ mobilization and contraction in airway smooth muscle. Am J Physiol Lung Cell Mol Physiol 2007; 293: L1406-L1418.

23 Fessel JP, Hamid R, Wittmann BM, et al. Metabolomic analysis of bone morphogenetic protein receptor type 2 mutations in human pulmonary endothelium reveals widespread metabolic reprogramming. Pulm Circ 2012; 2: 201-213.

24 Tibshirani R, Hastie T, Narasimhan B, et al. Diagnosis of multiple cancer types by shrunken centroids of gene expression. Proc Natl Acad Sci USA 2002; 99: 6567-6572.

25 Aggio RB, Ruggiero K, Villas-Bôas SG. Pathway Activity Profiling (PAPi): from the metabolite profile to the metabolic pathway activity. Bioinformatics 2010; 26: 2969-2976.

26 Metallo CM, Gameiro PA, Bell EL, et al. Reductive glutamine metabolism by IDH1 mediates lipogenesis under hypoxia. Nature 2012; 481: 380-384.

27 Conlon TM, Bartel J, Ballweg K, et al. Metabolomics screening identifies reduced L-carnitine to be associated with progressive emphysema. Clin Sci 2016: 130: 273-287.

28 Hyyti OM, Ledee D, Ning XH, et al. Aging impairs myocardial fatty acid and ketone oxidation and modifies cardiac functional and metabolic responses to insulin in mice. Am J Physiol Heart Circ Physiol 2010; 299: H868-H875.

29 Cloonan SM, Glass K, Laucho-Contreras ME, et al. Mitochondrial iron chelation ameliorates cigarette smoke-induced bronchitis and emphysema in mice. Nat Med 2016; 22: 163-174.

30 Boroughs LK, DeBerardinis RJ. Metabolic pathways promoting cancer cell survival and growth. Nat Cell Biol 2015; 17: 351-359.

31 Eng $\mathrm{CH}$, Abraham RT. The autophagy conundrum in cancer: influence of tumorigenic metabolic reprogramming Oncogene 2011; 30: 4687-4696.

32 Liu Y, Hyde AS, Simpson MA, et al. Emerging regulatory paradigms in glutathione metabolism. Adv Cancer Res 2014; 122: 69-101.

33 Chettimada S, Gupte R, Rawat D, et al. Hypoxia-induced glucose-6-phosphate dehydrogenase overexpression and -activation in pulmonary artery smooth muscle cells: implication in pulmonary hypertension. Am J Physiol Lung Cell Mol Physiol 2015; 308: L287-L300.

34 Li M, Riddle S, Zhang $\mathrm{H}$, et al. Metabolic reprogramming regulates the proliferative and inflammatory phenotype of adventitial fibroblasts in pulmonary hypertension through the transcriptional corepressor C-terminal binding protein-1. Circulation 2016; 134: 1105-1121.

35 Plecitá-Hlavatá L, Tauber J, Li M, et al. Constitutive reprogramming of fibroblast mitochondrial metabolism in pulmonary hypertension. Am J Respir Cell Mol Biol 2016; 55: 47-57.

36 Golpon HA, Coldren CD, Zamora MR, et al. Emphysema lung tissue gene expression profiling. Am J Respir Cell Mol Biol 2004; 31: 595-600. 
37 Ning W, Li CJ, Kaminski N, et al. Comprehensive gene expression profiles reveal pathways related to the pathogenesis of chronic obstructive pulmonary disease. Proc Natl Acad Sci USA 2004; 101: 14895-14900.

38 Pierrou S, Broberg P, O'Donnell RA, et al. Expression of genes involved in oxidative stress responses in airway epithelial cells of smokers with chronic obstructive pulmonary disease. Am J Respir Crit Care Med 2007; 175: 577-586.

39 Selak MA, Armour SM, MacKenzie ED, et al. Succinate links TCA cycle dysfunction to oncogenesis by inhibiting HIF- $\alpha$ prolyl hydroxylase. Cancer Cell 2005; 7: 77-85.

40 Finkel T. The metabolic regulation of aging. Nat Med 2015; 21: 1416-1423. 